\title{
Stress in special groups
}

Michael Sotiriou

From $1^{\text {st }}$ International Congress on Neurobiology and Clinical Psychopharmacology and European Psychiatric Association Conference on Treatment Guidance

Thessaloniki, Greece. 19-22 November 2009

Stress occurs when homeostasis is threatened or perceived to be so. The stress system with its central and peripheral effectors regulate the brain's cognitive, reward and fear systems, wake-sleep centers as well as the growth, reproductive and thyroid hormone axes, and influence the gastrointestinal, cardiorespiratory, metabolic and immune systems. Malfunction of the stress system might impair growth, development, behavior and metabolism, which potentially lead to various acute and chronic disorders.

The speech will be focused: a) On linguistics, where stress is the relative emphasis that may be given to certain syllables in a word and stress manifests itself in the speech stream are highly language dependent. b) Gender is an important biological determinant of vulnerability to psychosocial stress. Research studies focused on the sex differences in stress responses revealed individual differences in stress reactivity in addition to genetic, socio-cultural, hormonal and developmental factors. Women appear more physiologically reactive to social rejection challenges, but men react more to achievement challenges. Women's greater reactivity to rejection stress may contribute to the increased rates of affective disorders in women. Another study specifically indicates that women with the BDNF Val/ Met genotype and men with the $\mathrm{Val} / \mathrm{Val}$ may be particularly vulnerable to social stress mediated by brain stress system activity (Psychoneuroendocrinology, 2009). c) Poor self-concept, impatience, poor consequential thinking, inability to set realistic goals and to prioritize, poor resource management, poor health habits, overdependence, inflexibility are some of personality-related stressors. d) Stress responses associated with possible implications for psychopathology during childhood, adolescents and older adults. Prenatal stress and maternal exposure to exogenous glucocorticoids can lead to permanent modification of hypothalamo-pituitary-adrenal function and stress-related behaviour. Childhood stress and trauma have been related to adult psychopathology in different psychiatric disorders. e) Some transitions and dynamics can lead families to excessive stress. There are many stresses in healthy and dysfunctional families. Research revealed that secure-base interactions between parents and adolescents facilitate physiological regulation of stress, especially for adolescents with externalising symptomatology. f) Finally, the effects of stress reactivity may lead to physical symptoms and disorders (e.g. hypertension, appetite and gastrointestinal problems, skeletal muscles increased tension, smooth muscles increased contraction, visceral obesity, hyperlipidemia, hyperglycemia, cardiovascular disease, type II diabetes, suppression of immune system). There is also a link between stress and fertility. Women with infertility report equivalent levels of anxiety and depression as women with cancer, HIV status, and heart disease. Men also suffer fertility related stress, though they frequently keep their anxiety to themselves for fear of adding to their partner's burden.

Published: 22 April 2010

doi:10.1186/1744-859X-9-S1-S40

Cite this article as: Sotiriou: Stress in special groups. Annals of General

Psychiatry 2010 9(Suppl 1):S40.

Psychiatric Adult Unit, Kavala General Hospital, NHS, Kavala, Greece 